\title{
Intratympanic Dexamethasone Injection in Chronic Tinnitus Associated with SNHL
}

\author{
AYAT A. ABU-ELNASR, M.D.*; HODA E. ALI, M.D.* and AMAL H. EWIDA, M.D.** \\ The Departments of Otorhinolaryngology* and Audiology**, Faculty of Medicine, Al-Azhar University, Cairo, Egypt
}

\begin{abstract}
Background: Tinnitus is a common distressing complain characterized by perceived sensation of sound without an external stimulus. Intra-tympanic (IT) dexamethasone injection is used for the treatment of tinnitus associated with sensory neural hearing loss (SNHL) by introducing dexamethasone through the tympanic membrane, leading to decreasing systemic toxicity and a higher perilymph steroid level.
\end{abstract}

Aim of the Study: The aim of this study was to express our experience in evaluation the effect of IT dexamethasone injection for controlling idiopathic chronic tinnitus of cochlear origin associated with SNHL.

Patients and Methods: A prospective interventional study of eighty patients with subjective idiopathic tinnitus associated with SNHL for at least 1 year were treated by IT injection of dexamethasone, once per week for 4 weeks. Improvement in tinnitus was assessed using pure-tone audiometry, speech audiometry, tinnitus matching test, and Tinnitus Handicap Inventory by comparing the results before and after therapy. Follow-up after the end of the four injections, 1 and 3 months later.

Results: Pure-tone audiometry did not show significant improvement. However, there was a significant improvement in speech audiometry, tinnitus loudness, and Tinnitus Handicap Inventory after the end of the four injections and in the subsequent evaluations 1 and 3 months later.

Conclusion: IT dexamethasone injection could be a simple and effective method for controlling subjective idiopathic chronic tinnitus. The tinnitus may not disappear, but could be markedly decreased and easily tolerated.

Key Words: IT injection - Tinnitus - (THI) Tinnitus Handicap Inventory - (SNHL)

\section{Introduction}

SUBJECTIVE idiopathic tinnitus is defined as the aberrant perception of noise or sound without any external stimulation [1]. Subjective tinnitus is very common and is heart only by the patient. It may arise due to electrophysiological disturbance

Correspondence to: Dr. Ayat A. Abu-Elnasr,

The Department of Otorhinolaryngology, Faculty of Medicine Al-Azhar University, Cairo, Egypt anywhere in the auditory system: The external ear canal, tympanic membrane, ossicles, cochlea, auditory nerve, brainstem or cerebral cortex [2].

There are no FDA-approved drugs to treat tinnitus [3], and few drugs reliably suppress or eliminate chronic tinnitus in the majority of patients. The lack of drug therapies is due to many factors as inadequate information about biological basis of tinnitus, the heterogeneity of the tinnitus population, the wide range of medical causes of tinnitus, and the huge cost of drugs that specifically treat tinnitus [4].

The (IT) intratympanic treatment of inner ear disorders has increased over the past two decades. The Principal advantages of The IT approach, in tinnitus therapy is the ability to deliver therapeutic concentrations of a pharmaceutical agent to the affected inner ear, with only minimal systemic exposure, thus avoiding side effects on the unaffected ear and/or the central nervous system [5]

Dexamethasone has anti-inflammatory properties. Compared with systemic administration, IT injection produces much higher intracochlear drug concentrations. Cortisol levels in the perilymph do not increase following intravenous administration of a high dose [6]. This is because the inner ear is an end organ with regards to its blood supply, and it is protected by a blood-labyrinth barrier [7] Dexamethasone is most widely used due to lack of the adverse reactions in other drugs (Methylprednisolone produces the highest relative concentration in lymphatic fluid, but it often provokes a burning sensation during injection. Hydrocortisone is reported to cause an inflammatory reaction of the round window membrane in a morphologic study with rates [1].

Sensorineural hearing loss (SNHL): SNHL represents $90 \%$ of hearing loss. It can be mild, 
moderate, severe, profound, or total. SNHL occurs as a consequence of damaged or deficient cochlear hair cell function during the lifetime of an individual. Presbycusis is an age-related deterioration of hearing ability, in elderly individuals [8]. Noise can also cause sensorineural hearing loss that increases over time. Loud noise damages the delicate structures in the ear due to trauma and accumulation of free radicals \& excess glutamate, as well as altering intracellular magnesium and calcium levels [9]. Infections and a condition called Meniere's disease can also lead to inner ear damage and sensorineural hearing loss [10]

The aim of this study was to express our experience in evaluating the effect of IT dexamethasone injection for controlling chronic idiopathic tinnitus of cochlear origin associated with SNHL.

\section{Patients and Methods}

Prospective interventional study was carried out on eighty patients having persistent subjective tinnitus associated with variable degree of SNHL of cochlear origin for at least 1 year, refractory to medical line of management, in the age group of 30-60 years were selected randomly from the ENT and Audiology outpatient clinics at Al-Azher University Hospitals, Egypt. The study was conducted from April 2014 to March 2017.

The study protocol was approved by the local ethics committee. Informed consent was obtained from all participants. Tinnitus may be unilateral or bilateral, the ear with more annoying and louder tinnitus was chosen. Patients with a history of trauma, otitis media, otologic surgery, ototoxic drug intake, noise-induced hearing loss, Meniere's disease, systemic illness, and neurologic illness were excluded from the study. A detailed history was taken from all patients.

Otoscopic examination and neurologic examination were also carried out, after which they underwent a battery of tests.

\section{Audiological assessment:}

- Pure-tone audiometry was performed using Puretone audiometer interacoustics AC40 with head phones TDH 39 and bone vibrator B71. Sound treated room of controlled acoustical room (iac) at frequencies between 0.25 and $8.0 \mathrm{kHz}$ for air conduction and between 0.5 and $4.0 \mathrm{kHz}$ for bone conduction. The four-frequency pure-tone average (4-PTA) for air conduction was calculated at frequencies of $0.5,1.0,2.0$, and $4.0 \mathrm{kHz}$.

- Speech audiometry was evaluated by measurements of speech reception threshold using Arabic spondee word List [11] and speech discrimination score was determined using Arabic phonetically balanced word [12] for each ear separately.

- Impedance measurements were taken, including tympanometry and acoustic reflex testing.

- Auditory brainstem response was evaluated when a retro-cochlear lesion was suspected to be excluded.

Tinnitus matching test [13]: The tinnitus matching test was carried out with an audiometer Madsen (Madsen company, Denmark) OB 822 and included the following:

1- Pitch matching was measured by presenting two different frequencies of pure tone or narrow band noise, and the patient was asked to judge whether the pitch of the first or the second of the two successive tones is closer to the tinnitus the patient hears. The tone is presented to the contra lateral ear with successive approximations until the most dominant frequency has been identified.

2- Loudness matching was measured by presenting the external sound previously identified during pitch matching to the ear contra lateral to the tinnitus and was increased or decreased in intensity until the patient judges that the loudness is equal to that of his or her tinnitus. The test for the intensity match is delivered in $1 \mathrm{~dB}$ steps.

3- Minimal masking level was ascertained by determining the minimum level of white noise needed to achieve masking of the ongoing tinnitus. This test was performed monaurally in the affected ear in $1 \mathrm{~dB}$ steps. The test was stopped immediately if the stimulus became uncomfortably loud.

4- Residual inhibition was carried out by presenting a white noise to the affected ear at $10 \mathrm{~dB}$ above the minimum masking level for 60s. At the end of $1 \mathrm{~min}$, the patient assesses whether the tinnitus is gone, diminished, unchanged, or louder. A complete residual inhibition was referred to if the tinnitus was completely absent after a 1min exposure to the white noise; a partial residual inhibition was referred to if the tinnitus reduced but was not completely absent for a period of time; and negative residual inhibition was referred to if there was no change in tinnitus loudness.

Tinnitus Handicap Inventory: The THI is a selfadministered questionnaire to evaluate the impact of tinnitus on the quality of life. The patients responded to a specific tinnitus questionnaire [14] 25-item questionnaire that is scored on a three- 
point scale (no=0, sometimes $=2$, and yes $=4$ ). On the basis of the total THI score, tinnitus patients can be classified into five categories denoting handicap severity: Slight handicap (0-16), mild handicap (18-36), moderate handicap (38-56), severe handicap (58-76), or catastrophic (78-100) [15].

The patients were given a specific tinnitus questionnaire regarding: tinnitus duration, ear affected, subjective hearing loss, description of the sound heard and known otologic diseases/ previous treatments. The patients were then asked to indicate the intensity of tinnitus on a visual analog scale graded from 1 to 10 (1 was low and 10 was an unbearable level of intensity). Informed consent was obtained from all patients $[\mathbf{1 5 , 1 6}]$.

IT injection of dexamethasone: One ear of each of the 80 patients was treated with IT dexamethasone $(8 \mathrm{mg} / \mathrm{ml})$ injection under local anesthesia [15]. Actually, about $5 \mu 1$ is usually sufficient to fill the entire round window niche [17]. it is much more important how long a drug remains in the round window niche and can diffuse into the cochlea than the volume that is injected into the middle ear [18] to $1 \mathrm{ml}[19]$. An injection volume of $0.2-0.3 \mathrm{ml}$ is probably fully sufficient to cover the target area any additional quantities are likely to increase the sensation of pressure and possibly, pain in the middle ear, and they would spill back into the ear canal or drain off via the Eustachian tube [20].

Following a brief explanation of the procedure to the patient, otoscopy of the ear canal and tympanic membrane is performed. Excessive earwax or debris is cleared. A local anesthetic applied. Usually, EMLA cream (eutectic lidocaine/prilocaine mixture) xylocaine (4\% lidocaine solution or $10 \%$ pump spray), The xylocaine pump spray has a very short induction time of about 1-3 minute. EMLA cream requires $1 \mathrm{~h}$. Once the anaesthesia has taken effect, any remaining anaesthetic needs to be suctioned off prior to the injection to avoid spillage into the middle ear, and avoid vertigo or dizziness. During all these preparations, the drug can be warmed up to about body temperature to prevent caloric vertigo [5].

Under using a microscope with the patient sitting on the examination chair. For the procedure, the patient's head is usually placed in a position tilted $45^{\circ}$ towards the unaffected ear. This allows for the RWM to be at the lowest point of the middleear cavity, and the study medication to collect there and be in physical contact with the membrane. Patients are asked to remain in this position for about 30min [15] allowing the active substance to diffuse into the inner ear. In order to avoid loss of medication into the nasopharynx through the Eustachian tube, patients are also asked not to swallow, yawn, sneeze or speak [21].

For the injection, the RWM provides an attractive gateway to the inner ear., Typically, $1 \mathrm{ml}$ syringes are used. Preferably, a short bevel needle is used or, even better, one that is blunt. The injection is frequently performed via the posterior-inferior quadrant of the tympanic membrane, i.e., the area overlying the round window niche left ear: About 3 o'clock, right ear: About 9 o'clock, the injection may be performed antero-superior, the tympanic membrane is punctured directly with the needle (tympanopunction) and the injection is performed right away [5].

A drug with a target site of action inside the inner ear is injected into the middle-ear cavity, from where the active substance diffuses into the cochlea (Fig. 1). Diffusion occurs across the semipermeable round window membrane (RWM), driven by the concentration gradient between the middle ear and the perilymph-filled scala tympani on the opposite side of the RWM [5].

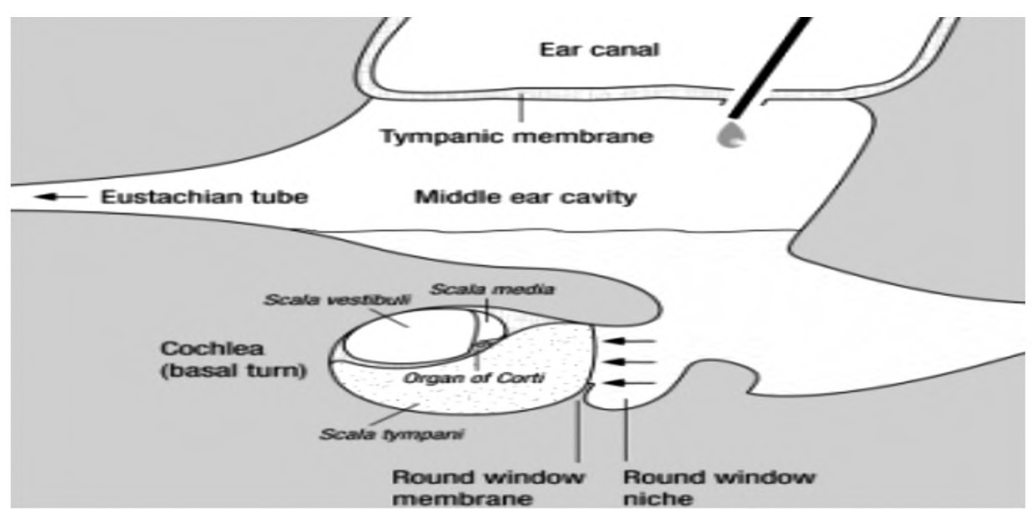

Fig. (1): Basic concept of intratympanic treatment. Medication is injected through the tympanic membrane into the middle ear cavity in a quantity that is sufficient to fill the round window niche. The active substance is diffusing across the round window membrane into the basal turn of the perilymph filled scala tympani of the cochlea from where it is spreading further (Meyer, 2013). 
The patients were acupunctured 4-5 times in the whole course of treatment [5]. Four injections were administered once per week for 4 weeks. Follow-up by Pure-tone audiometry, speech audiometry, tinnitus matching and THI were repeated after the end of the four injections, 1 and 3 months later [15]

Statistical design: Categorical variables were described as number and percentage and continuous variables were described as mean and SD (mean, $\mathrm{SD}$ ). Comparison between continuous variables was done using $t$-test and analysis of variance. $p$ value less than 0.05 was considered statistically significant.

\section{Results}

The mean age of patients was $40 \pm 11$, with an age range of $30-50$ years. The study included 22 male and 58 female patients. The duration of tinnitus ranged from 12 months to 18 months with a mean of $13.7 \pm 10.0$. The tinnitus was bilateral in 52 patients and unilateral in 28 patients.

There was no significant difference in the mean pure-tone thresholds after dexamethasone injection in tinnitus patients, $9 p=0.776$ ). There was a signif- icant improvement in speech discrimination score after therapy ( $p=0.038$ ) (Table 1, Fig. 2 ).

There was a significant reduction in tinnitus loudness level when measured subjectively, using tinnitus matching tests. Moreover, the minimum masking level was reduced significantly when comparing pre-therapy and post-therapy levels (Tables 2,3). A total of $74(92.5 \%)$ tinnitus patients showed reduction in their loudness level and minimum masking level.

As regards residual inhibition (RI), only three patients changed from negative RI to partial RI; no patient changed to complete RI. There was no statistically significant difference between preinjection and post-injection RI ( $p=0.84)$.

The results of THI showed statistically significant improvement after dexamethasone injection. There was also an improvement in patients at the end of the therapy and subsequent evaluations (1 and 3 months later), but this improvement was not statistically significant (Table 4).

Before injection, tinnitus patients had mainly severe or catastrophic handicap, but their scores reduced significantly after therapy to slight, mild, or moderate handicap ( $p=0.001)$ (Fig. 3).

Table (1): Mean, SD, and P level of pure-tone average, and speech discrimination score for the study samples before and after dexamethasone injection.

\begin{tabular}{lccccc}
\hline \multirow{2}{*}{$\begin{array}{l}\text { Audiometric } \\
\text { test }\end{array}$} & $\begin{array}{c}\text { Mefore } \\
\text { injection }\end{array}$ & $\begin{array}{c}\text { First } \\
\text { evaluation }\end{array}$ & $\begin{array}{c}\text { Second } \\
\text { evaluation }\end{array}$ & $\begin{array}{c}\text { Last } \\
\text { evaluation }\end{array}$ & $p$-value \\
\cline { 2 - 6 } PTA & $44.1 \pm 20.5$ & $42.5 \pm 20.1$ & $43.1 \pm 19$ & $43 \pm 19.1$ & 0.776 \\
SD & $80.7 \pm 16.3$ & $88.9 \pm 6.7$ & $85.1 \pm 13.4$ & $86.2 \pm 11.3$ & $0.038^{*}$ \\
\hline
\end{tabular}

ANOVA test used to obtain.

Value: ANOVA analysis of variance.

PTA: Pure tone average.

NS: no significant difference $(p>0.05)$, significant difference $(p<0.05)$

Table (2): The mean and SD of loudness level of the study samples before and after dexamethasone injection.

\begin{tabular}{lcc}
\hline & $\begin{array}{c}\text { Loudness level } \\
(\text { Mean } \pm \text { SD) }\end{array}$ & $p$-value \\
\hline Base line & $50.2 \pm 12.4$ & \\
First evaluation & $40.1 \pm 12.8$ & 0.131 \\
Second evaluation & $38.1 \pm 18.4$ & $0.014^{*}$ \\
Last evaluation & $34.9 \pm 14.6$ & $0.003^{*}$ \\
\hline
\end{tabular}

Table (3): The mean and SD of minimal masking level (MML) of the study samples before and after dexamethasone injection.

\begin{tabular}{lcc}
\hline & $\begin{array}{c}\text { Minimal masking level } \\
(\text { Mean } \pm \text { SD })\end{array}$ & $p$-value \\
\hline Base line & $60.5 \pm 18.1$ & \\
First evaluation & $53.7 \pm 13.5$ & $p=0.139$ \\
Second evaluation & $50.2 \pm 11.3$ & $p=0.023^{*}$ \\
Last evaluation & $34.9 \pm 16.6$ & $p=0.001^{*}$ \\
\hline
\end{tabular}


Table (4): Mean, SD, and $p$ level of Tinnitus Handicap Inventory for the study samples before and after dexamethasone injection.

\begin{tabular}{lccccc}
\hline TH I & Range & Mean $\pm \mathrm{SD}$ & $p_{1}$ & $p_{2}$ & $p_{3}$ \\
\hline Before injection & $30-80$ & $60.1 \pm 11.2$ & & & \\
First evaluation & $14-60$ & $40.3 \pm 12.7$ & $0.001^{*}$ & & \\
Second evaluation & $14-62$ & $38.8 \pm 14.6$ & $0.001^{*}$ & 0.411 & \\
lastevaluation & $14-60$ & $36.9 \pm 13.3$ & $0.001^{*}$ & 0.121 & 0.422 \\
\hline
\end{tabular}

TH I: Tinnitus Handicap Inventory.

$p_{1}$ : Comparison between base line and each one of other evaluations using $t$-test.

$p 2$ : Comparison between first evaluations and each one of other evaluations using $t$-test.

$p_{3}$ : Comparison between second evaluations and each one of other evaluations using $t$-test.

$p_{3}$ : Comparison between third evaluation and each one of other evaluations using ANOVA test.

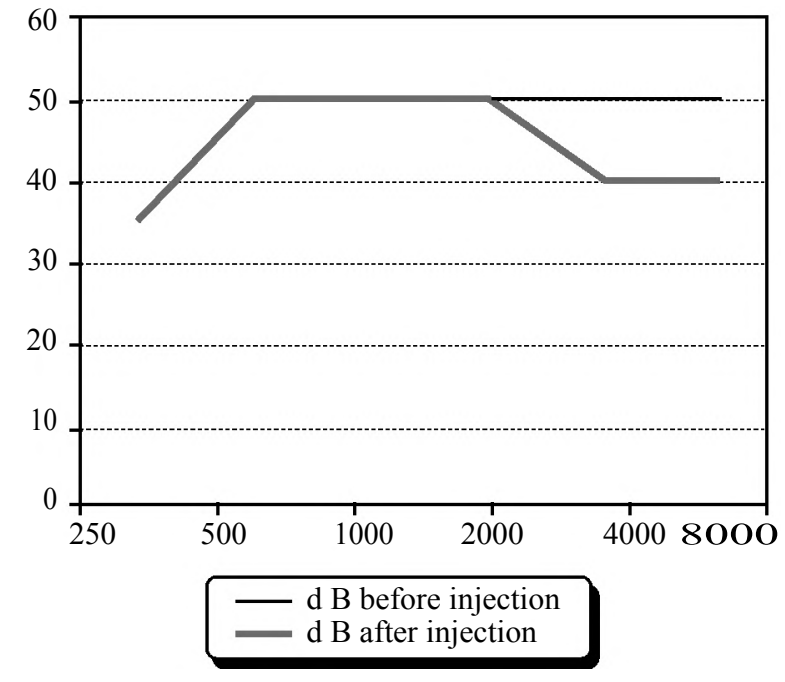

Fig. (2): The mean of pure-tone thresholds at different frequencies before and after dexamethasone injection in tinnitus patients.

\section{Discussion}

IT steroids may have an anti-inflammatory effect in the labyrinth, especially beneficial response in inner ear diseases with likely immune causes [22]. Other mechanism of action for IT dexamethazone injection in tinnitus patients, suggest that steroid perfusion of labyrinthine tissues can affect sodium and fluid transport in vitro studies [23]. The choice of an IT route in the treatment of tinnitus has two advantages: First, high perilymph levels as a result of providing a direct passage through the oval window membrane, second, adverse effects of systemic administration of the drug are avoided [24]

In the present study, no significant changes in PTA, but SD\% significantly improved. Weshahy et al., [15] study agreed with our results, In which IT dexamethasone injection in 30 patients with nearly the same inclusion and exclusion criteria and reported no significant difference between pretherapy and post-therapy levels but there was a

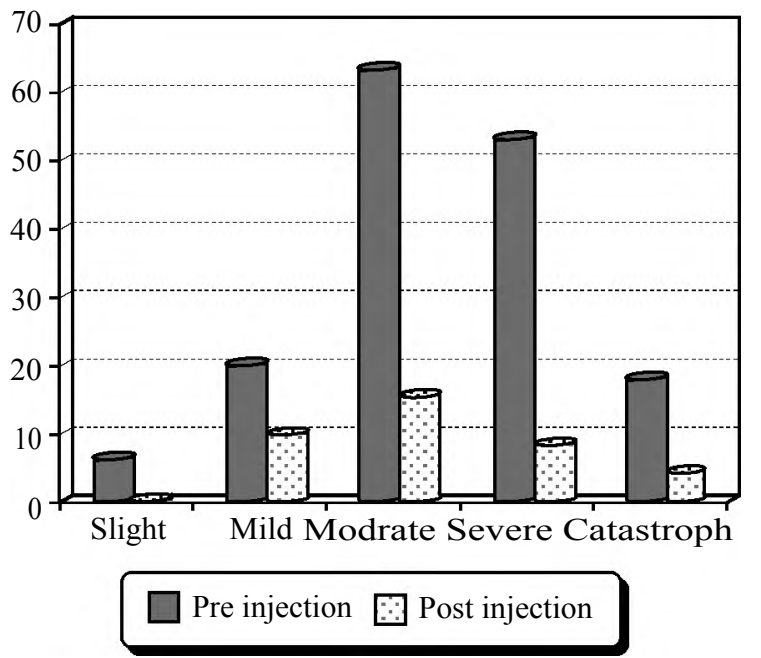

Fig. (3): Total Tinnitus Handicap Inventory (THI) score of the study samples before and after dexamethasone injection.

significant improvement in speech discrimination score after therapy. Our results were also in agreement with those of Parelkar et al., [22]. Who reported no change in the hearing levels before and after treatment but speech discrimination show (30$60 \%$ ) improvement that has been associated with IT dexamethasone.

In this study, the tinnitus loudness level and minimal masking level reduced significantly on tinnitus matching test evaluation after IT dexamethasone injection, with improvement of these parameters in $74(92.5 \%)$ patients. These results are in agreement with Mahmoud and Hafize [25], who reported a significant difference between the study group and the control group (tinnitus patients without IT dexamethasone injection) for the two parameters, loudness level and minimal masking level, and concluded that the tinnitus matching test could be used as a subjective evaluation for tinnitus and outcome measure of the therapy. In contrast to Faramarz and Fatemeh [26] who studied IT 
dexamethasone injection in 100 patients with nearly the same inclusion and exclusion criteria and reported different results as Seventy-three of 100 patients had abnormal SDS, with improvement in SDS observed in 26 (35\%) of these cases. Eightyseven of 100 patients complained of tinnitus, while $49(57 \%)$ of these patients showed improvement in their tinnitus score. Tinnitus score was not worsened in any patient. The mean values of PTA, SDS, and tinnitus score before treatment were 44 $\mathrm{dB}, 77 \%$ and 40 respectively; changing to $37 \mathrm{~dB}$, $89 \%$ and 31 , respectively following treatment $\left(p<{ }^{0} \cdot{ }^{05}\right)$.

In this study THI scores were also improved significantly after IT injection. Most of the patients changed from severe and catastrophic handicap to slight, mild, and moderate handicap; only nine of the tinnitus patients showed full recovery of tinnitus. Our results are in agreement with the studies of Shulman and Goldstein [27] that treated 10 patients having tinnitus with intra-tympanic dexamethasone injections. Five patients had tinnitus control for at least 1 year and 2 had tinnitus control for only a few hours. Three patients showed no improvement. Sakata et al., [28] used IT dexamethasone injection in tinnitus and reported recoveries of 71,70 , and $74 \%$ respectively.

In 2002, Cesarani et al., [29] described 54 patients treated with intra-tympanic dexamethasone injections .Of these patients, $34 \%$ showed complete resolution of tinnitus, $40 \%$ experienced significant improvement, and $26 \%$ experienced no change. The reported $34 \%$ full recovery of the tinnitus. This could be attributed to the difference in the inclusion criteria of their study group in which the duration of tinnitus did not exceed 3 months. An et al., [30] also reported that Tinnitus was cured in 43 of 114 patients (37.7\%) within 3 months. The mean tinnitus loudness score, the mean tinnitus awareness score, and the mean THI scores were significantly reduced at 3 months after a IT dexamethasone injection.

In contrast, Araujo et al., [31] studied the effectiveness of IT dexamethasone injection as a treatment for severely disabling cochlear tinnitus and they concluded that there was no advantage of dexamethasone over saline solution and both solutions produced a placebo-like improvement in about $30-40 \%$ of patients. They attributed their results to the inclusion of patients with severely disabling tinnitus, which is different from common forms of tinnitus; the symptoms are intense, with a high annoyance level and an affective component that renders the patient incapable of performing daily tasks efficiently. The central component of severely disabling tinnitus even when of cochlear origin makes the IT injections of dexamethasone inefficient in some group of patients, especially in those with severely disabling tinnitus [32].

In our study, no side effects except for transient injection-site pain in few patients were noted. Sufficient warming of the drug, the use of fine needles and appropriate local anesthesia, a gentle rate of injection, and avoidance of excessive injection volumes seem to be key factors for good local tolerance [33]. Stachler etal., [34] reported more local side effects including: Injection-site pain, dizziness, caloric vertigo, infection, persistent tympanic membrane perforation, or possible vasovagal or syncope episodes during injection.

Conclusion and recommrndation: IT dexamethasone injection could be a simple and effective method for controlling subjective idiopathic chronictinnitus associated with SNHL. The tinnitus could be alleviated to help the patient to cope more easily with the disease, decreasing their handicap and improving their quality of life.

One limitation of this study, a control groupwould not be ethically acceptable as a placebocontrolled study so controlled studies are recommended to confirm these results. Studying the effect of different therapeutic molecules on control of subjective idiopathic tinnitusassociated with SNHL is also recommended.

\section{References}

1- NONDAHL D., CRUICKSHANKS K., WILEY T., et al.: The 10-year Incidence of Tinnitus Among Older Adults. International Journal of Audiology, 49 (8): 580-585, 2010;.

2- SEIDMAN M.D.: Glutamate antagonists, steroids, and antioxidants as therapeutic options for hearing loss and tinnitus and the use of an inner ear drug delivery system. Int. Tinnitus. J., 4 (2): 148-54, 1998.

3- VIO M.M. and HOLME R.H.: Hearing loss and tinnitus: 250 million people and a US $\$ 10$ billion potential market. Drug Discov Today, 10: 1263-1265, 2005.

4- ROBERTS L.E., EGGERMONT J.J., CASPARY D.M., SHORE S.E., MELCHER J.R. and KALTENBACH J.A.: Ringing ears: The neuroscience of tinnitus. Journal of Neuroscience. 30 (45): 14972-9, 2010.

5- MEYER T.: Intratympanic treatment for tinnitus: A review. Noise Health, 15: 83-90, 2013.

6- NIEDERMEYER H.P., ZAHNEISEN G., LUPPA P., BUSCH R. and ARNOLD W.: Cortisol levels in the human perilymph after intravenous administration of prednisolone. Audiol. Neurootol., 8: 316-321, 2003. 
7- STEPP C.E. and VOSS S.E.: Acoustics of the human middle-ear air space. J. Acoust. Soc. Am., 118: 861-871, 2005.

8- HUANG Q. and TANG J.: Age-related hearing loss or presbycusis. Eur. Arch. Otorhinolaryngol., 267: 11791191, 2010.

9- ANN C.Y. WONG and ALLEN F.: Ryan. "Mechanisms of Sensorineural Cell Damage, Death and Survival in the Cochlea." Frontiers in Aging Neuroscience, 7 (7): 58, 2015.

10- LOPEZ-ESCAMEZ JOSE A., CAREY JOHN, CHUNG WON-HO. GOEBEL JOEL A. MAGNUSSON MÅNS. MANDALÀ MARCO. NEWMAN-TOKER DAVID E. STRUPP, MICHAEL and SUZUKI MAMORU.: Diagnostic criteria for Menière's disease. Journal of Vestibular Research: Equilibrium \& Orientation., 25 (1): 1-7, 2015.

11- SOLIMAN S., FATHALLA A. and SHEHATA W.: Development of Arabic suggested spondiac words test. Ain Shams Med. Congress., 2: 1220, 1985.

12- SOLIMAN S.: Speech discrimination audiometery using Arabic phonetically balanced words. Ain Shams Med. J., 27: 27-30, 1976.

13- SANDLIN R.E. and OLSSON R.T.: Subjective tinnitus: it's mechanism and treatment. In: Valenete M., Hosford H., Ross G.R., editors. Audiology treatment. New York, NY: Thieme, pp., 691-697, 2000.

14- SHIM H.J., SONG S.J., CHOI A.Y., HYUNG LEE R. and YOON S.W.: Comparison ofvarious treatment modalities for acute tinnitus. Laryngoscope, 121: 261925, 2011.

15- WESHAHY H.F., MOHAMED E.S., SALEM M.A. and HUSSEIN M.M.: Intratympanic injection of dexamethasone for controlling subjective idiopathic tinnitus. Egypt J. Otolaryngol., 31: 224-230, 2015.

16- MCCOMBE A., BAGULEY D., COLES R., MCKENNA L., MCKINNEY C. and WINDLE-TAYLOR P.: British Association of Otolaryngologists, Head and Neck Surgeons. Guidelines for the grading of tinnitus severity: the results of a working group commissioned by the British Association of Otolaryngologists, Head and Neck Surgeons, 1999. Clin. Otolaryngol. Allied. Sci., 26: 388-393, 2001.

17- MUEHLMEIER G., BIESINGER E. and MAIER H.: Safety of intratympanic injection of AM-101 in patients with acute inner ear tinnitus. Audiol. Neurootol., 16: 3889, 2011.

18- SALT A.N. and PLONTKE S.K.: Principles of local drug delivery to the inner ear. Audiology and Neurotology, 14 (6): 350-60, 2009.

19- RAUCH S.D., HALPIN C.F., ANTONELLI P.J., BABU S., CAREY J.P., GANTZ B.J., et al.: Oral vs intratympanic corticosteroid therapy for idiopathic sudden sensorineural hearing loss: A randomized trial. JAMA, 305: 2071-9, 2011.

20- BIRD P.A., BEGG E.J., ZHANG M., KEAST A.T., MURRAY D.P. and BALKANY T.J.: Intratympanic versus intravenous delivery of methylprednisolone to cochlear perilymph. Otol. Neurotol., 28: 1124-30, 2007.
21- HAYNES D.S., O'MALLEY M., COHEN S., WATFORD K. and LABADIE R.F.: Intratympanic dexamethasone for sudden sensorineural hearing loss after failure of systemic therapy. Laryngoscope, 117: 3-15, 2007.

22- PARELKAR K., THORAWADE V., JAGADE M., NAGLE S., NATARAJ R., PANDARE M., et al.: Intratympanic injections: An unsolved mystery. Int. J. Otolaryngol. Head Neck. Surg., 4: 124-132, 2015.

23- PONDUGULA S.R., SANNEMAN J.D., WANGEMANN P., MILHAUD P.G. and MARCUS D.C.: Glucocorticoids stimulate cation absorption by semicircular canal duct epithelium via epithelial sodium channel. American Journal Physiol. Renal. Physiol., 286: F1127-35, 2004.

24- CHANDRASEKHAR S.S., RUBINSTEIN R.Y., KWARTLER J.A., GATZ M., CONNELLY P.E., HUANG E. et al.: Dexamethasone Pharmacokinetics in the Inner Ear: Comparison of Route of Administration and Use of $\mathrm{Fa}-$ cilitating Agents. Otolaryngology-Head and Neck Surgery, 122: 521-528, 2000.

25- MAHMOUD E.A. and HAFIZE M.E.: Transtympanic dexamethazone treatment for control of subjective idiopathic tinnitus. El-minia Med. Bull., 15: 240-252, 2004

26- MEMARI F. and HASSANNIA F.: Effect of Intratympanic Dexamethasone on Controlling Tinnitus and Hearing loss in Meniere's Disease Iran. J. Otorhinolaryngol. Jul., 26 (76): 129-133, 2014.

27- SHULMAN A. and GOLDSTEIN B.: Intratympanic drug therapy with steroids for tinnitus control: A preliminary report. Int. Tinnitus. J., 6: 10-20, 2000.

28- SAKATA E., ITOH A. and ITOH Y.: Treatment of cochlear-tinnitus with dexamethasone infusion into the tympanic cavity. Int. Tinnitus. J., 2: 129-135, 1996.

29- CESARANI A., CAPOBIANCO S., SOI D., GIULIANO D.A. and ALP INI D.: Intratympanic dexamethasone treatment for control of subjective idiophatic tinnitus: our Clinical Experience. Int. Tinnitus. J., 8:11-113, 2002.

30- AN Y.H., YU K.K., KWAK M.Y., YOON S.W. and SHIM H.J.: Prognostic factors for the outcomes of intratympanic dexamethasone in the treatment of acute subjective tinnitus. Otol. Neurotol., 35: 1330-1337, 2014.

31- ARAÚJO M.F.S., OLIVEIRA C.A. and BAHMADJR F.M.: Intratympanic Dexamethasone Injections as a Treatment for Severe, Disabling Tinnitus. Arch Otolaryngol Head Neck. Surg., 131: 113-117, 2005.

32- HOUSE J.W.: Therapies for tinnitus. Am. J. Otol., 10: 163-165, 1989.

33- BELHASSEN S., SALIBA I.: Pain assessment of the intratympanic injections: A prospective comparative study. Eur. Arch. Otorhinolaryngol., 269: 2467-2473, 2012.

34- STACHLER R.J., CHANDRASEKHAR S.S., ARCHER, S.M., ROSENFELD R.M., SCHWARTZ S.R., BARRS D.M., et al.: Clinical Practice Guideline: Sudden Hearing Loss. Otolaryngology-Head and Neck Surgery, 146: S1S35, 2012. 


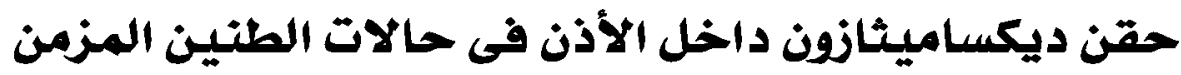

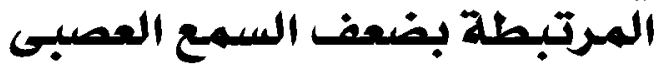

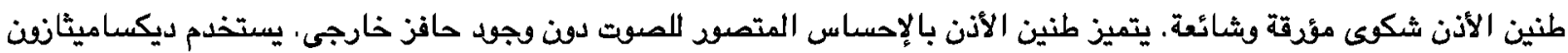

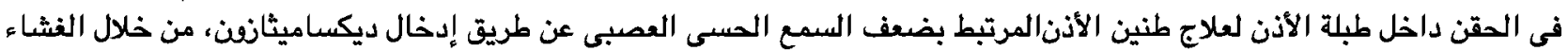

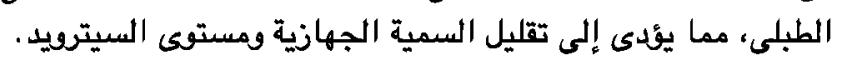

الهدف من الدراسة: كان الهدف من هذه الدراسة هو التعبير عن تجريتنا فى تقييم تأثير حقن ديكساميثانون داخل الأذن للسيطرة

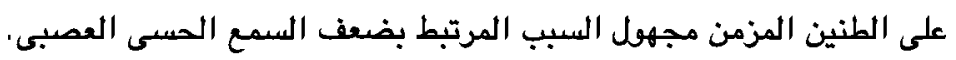

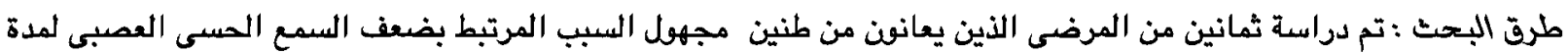

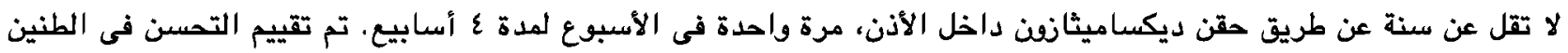

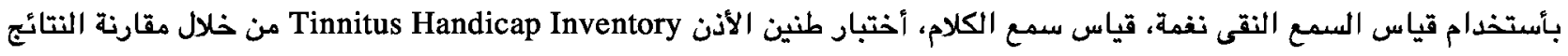
قبل وبعد العلاج. تمت المتابعة بعد نهاية الحقن الأريعة، ثم بعد شهر ثم بعد ثلاث أشهر أشهر فى وقت لاحق.

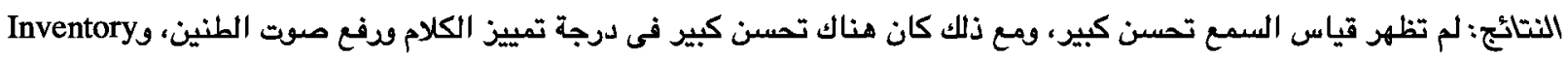
Tinnitus Handicap الخلاصة: يمكن أن يكون حقن ديكساميثانون داخل الأنن وسيلة بسيطة، وفعالة للسيطرة على طنين الأنن مجههل السبب. قد لا

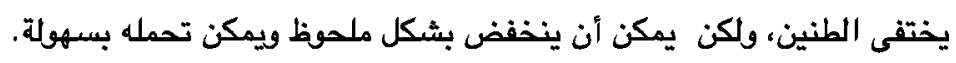

